\title{
The relational nature of rational numbers
}

Author:
Bruce Brown'
Affiliation:
'Education Department,
Rhodes University,
South Africa
Correspondence to:
Bruce Brown
Email:
b.brown@ru.ac.za
Postal address:
PO Box 94, Grahamstown
6140, South Africa
Dates:
Received: 17 July 2014
Accepted: 06 Apr. 2015
Published: 19 June 2015
How to cite this article:
Brown, B., 2015 , 'The
relational nature of rational
numbers', Pythagoras,
36(1), Art. \#273, 8 pages.
http://dx.doi.org/10.4102/
pythagoras.v36i1.273
Copyright:
C) 2015. The Authors.
Licensee: AOSIS
OpenJournals. This work is
licensed under the Creative
Commons Attribution
License.

It is commonly accepted that the knowledge and learning of rational numbers is more complex than that of the whole number field. This complexity includes the broader range of application of rational numbers, the increased level of technical complexity in the mathematical structure and symbol systems of this field and the more complex nature of many conceptual properties of the rational number field. Research on rational number learning is divided as to whether children's difficulties in learning rational numbers arise only from the increased complexity or also include elements of conceptual change. This article argues for a fundamental conceptual difference between whole and rational numbers. It develops the position that rational numbers are fundamentally relational in nature and that the move from absolute counts to relative comparisons leads to a further level of abstraction in our understanding of number and quantity. The argument is based on a number of qualitative, in-depth research projects with children and adults. These research projects indicated the importance of such a relational understanding in both the learning and teaching of rational numbers, as well as in adult representations of rational numbers on the number line. Acknowledgement of such a conceptual change could have important consequences for the teaching and learning of rational numbers.

\section{Introduction}

The 21st century has seen a resurgence of research, in both mathematics education and cognitive psychology, into the teaching, learning and understanding of rational numbers. A number of different approaches are taken in this research. Some emphasise the unity between whole and rational numbers, based on the observation that both represent magnitudes: quantities that can be represented by a point on a number line (Siegler, Thompson \& Schneider, 2011). They advocate ways of teaching that build on this unifying property. Others, such as privileged domain theories (Gelman \& Williams, 1998) and conceptual change theories (Ni \& Zhou, 2005), emphasise the discontinuity between rational number concepts and whole number concepts and investigate the conflicts these introduce into rational number learning. In particular, Vanvakoussi and Vosniadou (2004) and McMullen, Laakkonen, Hannula-Sormunen and Lehtinen (2015) have identified a 'whole number bias' in peoples' responses, when comparing the magnitudes of two rational numbers.

With regard to prior knowledge, it is generally accepted that rational number learning builds on a foundation of whole number understanding. Additional prior knowledge that is important for rational number learning but is not directly related to whole number understanding has been identified by McMullen, Hannula-Sormunen and Lehtinen (2013, 2014). This involves a comparison relational scheme, which provides a relational foundation for rational number understanding.

This article seeks to identify the fundamental nature of the discontinuity between whole and rational number concepts and relate this to the unifying conception of magnitude on a number line. It advances the argument that the relational property, as well is being important for the early teaching and learning of rational numbers, is also important for rational number understanding of Senior Phase and Further Education and Training learners and of adults. Furthermore, the relational property introduces a fundamental conceptual difference between whole number and rational number quantities. This difference involves a change from a quantity as an absolute count, to a quantity as a relative comparison. In addition, there is a corresponding increase in the level of abstraction of the concept of quantity.

\section{Transitions from whole to rational number understanding}

In the literature of both cognitive psychology and mathematics education, it is acknowledged that the development of rational number understanding involves a number of changes in the child's experience related to numbers. These may be viewed in different ways and this section will start with a brief review of these different approaches in the literature. 
The changes in children's experience result in the development of more complex operational schemata for numbers. Wright (2014) includes operations such as unitising (including equal subdivision of wholes into part units and consolidation of collections of wholes into grouped units), iteration, rational stretching and shrinking, partitioning and allocation. These operations allow a number of functionally different conceptual structures relating to rational numbers, identified by Kieren $(1976,1988)$ and Behr, Lesh, Post and Silver (1983). The structures are characterised as the partwhole, measurement, quotient, operator and ratio subconstructs. In addition, the child develops more complex numerical representations, such as fraction and decimal notation, and a more complex relation between symbol and number, seen in the multiple fraction representations of the same rational number.

The conceptual change orientation to rational number learning (McMullen et al. 2015; Ni \& Zhou, 2005; Vanvakoussi \& Vosniadou, 2004) identifies and investigates a number of differences in the properties of whole and rational numbers. Many of these are properties of whole numbers, that are not properties of rational numbers. Examples (Siegler, Fazio, Bailey \& Zhou, 2013) are: each whole number may be separated from the others by an open interval, the product of two whole numbers is generally greater than each and the whole numbers can be counted in their natural order. Two differences that have become an important focus of conceptual change research are the density of the rational numbers in the real numbers and the complexity of the process of ordering and comparing two rational numbers.

According to Vanvakoussi and Vosniadou (2004) and McMullen et al. (2015), responses when comparing the magnitudes of two rational numbers appear to be related to the manner in which these numbers are represented symbolically as fractions or decimals, as well as to the distance between their magnitudes on the number line. It appears as if the use of two whole numbers to represent fractions may have an important influence on comparisons of their magnitudes, a property termed the 'whole number bias'. The child's earlier development of whole number knowledge thus has an important influence on their developing rational number concept and this influence extends into the developed understanding of adults. The privileged prior nature of whole number learning is thus held to interfere with later rational number learning, a view that is shared by privileged domain theories (Gelman \& Williams, 1998).

Whole number knowledge does not appear to be the only prior knowledge of importance for rational number learning. The work of McMullen et al. $(2013,2014)$ indicates that a cognitive comparison relational scheme, which is not directly related to whole number knowledge, contributes an additional foundation for rational number learning. The power of using such relational understanding for early formal rational number learning has been demonstrated by Cortina, Visnovska and Zuniga (2014). They showed that the capacity to view the measurement unit and the quantity to be measured as separate entities contributed greatly to learning the inverse ordering relationship for unit fractions.

The integrated theory of Siegler et al. (2011) challenges the view that whole number learning interferes with fraction learning. Siegler et al. maintain that the property of magnitude is the 'only property that unites real numbers' (Siegler et al., 2013) and emphasise the importance of basing rational number teaching on the concept of magnitude. In this approach, the number line is used as a representational model for the conception of rational numbers as magnitudes. This model emphasises the one-dimensional, ordered nature of the number system. A teaching approach based on magnitude rather than the standard part-whole model has been researched by Moss and Case (1999) and has shown positive improvements in learning.

Each of these views on rational number learning offers important insights for the teaching and learning process. But taken together they may be read as conflicting and contradictory. This article advances the view that relational understanding provides a unifying perspective on these different views. It argues that conceptual change is indeed important in the learning transition from whole number to rational number thinking and that this discontinuity stems from the nature of the rational number concept. The interference of whole number properties in rational number learning then derives from the fundamental change in the nature of the concept of quantity, from absolute count to relative comparison. This change is brought on by incorporating the relational nature of rational numbers into the child's thinking about numbers. Finally, this article supports the opinion that the property of magnitude unites whole and rational numbers, but it will argue that the concept of magnitude, which allows this unity, relies on a relational interpretation of number. The achievement of this unity will thus occur with the successful negotiation of this fundamental transition in rational number learning.

\section{The research projects}

This article draws from four exploratory research projects investigating rational number teaching and learning at different levels: in Grade R, Grade 3, Grades 4-7 and with teachers and student teachers. The overarching focus of these research projects was to investigate the manner in which teachers and children made meaning of rational numbers and the ways in which teaching influenced the meaning that children made of rational numbers. Each project focused on a different question relating to this issue. In the Grade $\mathrm{R}$ project, ways of developing Grade R children's foundational knowledge for rational number learning were explored. The Grade 3 research focused on learners' everyday knowledge relating to rational number learning. In Grades 4-7, ways of teaching and learning rational numbers were investigated in a school. The fourth project focused on student teachers' and teachers' knowledge of rational numbers. The primary focus of this article, the fundamental nature of the 
discontinuity between whole and rational number concepts and the corresponding transition in teaching and learning, emerged as an important concern during the course of these projects. These were all small-scale, qualitative research projects. The first three involved English-medium primary or pre-schools in the Eastern Cape. The schools were all historically advantaged, although the first had an even mix of children from historically advantaged and disadvantaged backgrounds. This article will discuss the whole numberrational number discontinuity, drawing on episodes from these projects to illustrate the emergence of this concern.

\section{Rational numbers as relations Relational comparisons in the common introduction to rational numbers}

It is still common practice (Kilpatrick, Swafford \& Swindell, 2001; Lamon, 2007) for children to be introduced to rational numbers by working with parts of wholes. Fractions are then used to quantify the 'size' of the part in relation to the 'size' of the whole. In this way, the child experiences particular instances of rational numbers describing parts of objects, such as half of an apple, a third of a cake and even three-quarters of a slice of bread. By extensive repetition, it is expected that the child will abstract this descriptive relationship to form a conception of a rational number, in much the same fashion that earlier they abstracted the concept of a counting (whole) number from multiple instances of counts of objects. Concrete or drawn objects are soon supplanted by partially shaded diagrams that the child learns to describe using partwhole 'double counts'.

\section{Prior learning and rational comparisons}

The research of McMullen et al. $(2013,2014)$ indicates the importance of the scheme of relational comparison as prior knowledge for the early learning of rational numbers. Such a scheme was evident in both the Grade R and the Grade 3 research. In the Grade 3 project, in-depth clinical interviews with two teachers and two learners were carried out to explore children's everyday knowledge for rational numbers. In one task, children were asked to allocate dough to containers of different sizes for cooking. They both competently carried out this task, relating the relative sizes of the containers to the relative sizes of the dough. But neither child was able to describe what they had done using quantities or relational terms; the most that they could offer was that one was bigger than the other.

The Grade R investigation reported in this article involved a number of clinical interviews with four children, each of whom could count confidently from $0-50$. A number of tasks were formulated that may contribute to the development of the children's relational capacity. Children were asked to carry out the tasks and their engagement with the rational relation was investigated. The first task involved collections of easily identifiable objects, such as models of people and drawings of bicycles and tricycles. For each collection, children were asked to count the objects in the collection and also to count the number of specific components of the objects in the collection (arms for people, wheels for bicycles and tricycles). Toy models were presented for the collections of people and pictures of bicycles and tricycles were shown. The tasks for each object were presented in sequence, starting with a single object, then presenting collections with more and more objects. Finally a number of objects was specified (without a physical or diagrammatical presentation) and the child was asked to work out how many components would be in a collection with that number of objects. Firstly, no help was given for the final task, but if little progress was made, the child was encouraged to draw.

For each sequence, the children confidently counted wholes and components. Also, they stated how many components were included in each whole. For example, two arms for each person. When asked to compare the counts of wholes and components, they would only state that there were more components than wholes, and if more detail was requested, they reiterated the relationship for each whole. In the final task of the sequence, they were not able to determine the number of components without a drawing, but they were able to make an appropriate drawing and correctly count the components from this. In a further task sequence, the children were asked to count handles and wheels of a number of tricycles. In this case, they were not able to formulate the relationship of 2 handles to 3 wheels (even for a single tricycle) and also they found great difficulty in drawing a collection with a given number of handles.

It appeared as if the children were working with a constitutive object-component relationship for each object (how many arms were needed to form a person), rather than a numerical relationship between quantities. For when asked to describe the relationship, they either gave the number of components for each whole or they stated that there were more components than wholes. And without prompting, they did not give the total number of objects and components for comparison. In the final sequence, the only response was that there were more wheels than handles, showing some difficulty in working with component-component relationships.

In subsequent tasks, drawings of scoops of ice cream in different containers were used. Firstly, the children were shown a drawing of a single container and asked to count the number of scoops in this container. Then they were shown pictures of different numbers of containers and asked how many scoops of ice cream would be needed for the containers. Children worked with three sequences, first with cones (2 scoops per cone), then cups (3 scoops per cup) and lastly bowls (6 scoops per bowl). Each child was able to identify and describe the relation for each container (each cone has 2, each cup has 3 and each bowl has 6). For each sequence, they were then shown a single empty container and then two empty containers. They all needed some help with these first two counts (in the form of the researcher verbally reiterating the number of scoops per container shown by the first picture) as they became familiar with the task. They generally worked by indicating empty containers with their fingers and counting 
finger taps for each ice cream scoop in the container. Having successfully completing the two-container task, they were all able to confidently and quickly count how many scoops were needed in different numbers of cones or cups, showing an effective use of the 1-2 and 1-3 relation. For the bowl, they found it difficult to count finger taps, tending to lose count and so count either more or less than six for some bowls, but they could confidently draw and then count the final result.

The children did not use fractions to describe what they were doing. They also did not describe the relationships between their counts of objects and components (or containers and ice cream scoops) in multiplicative terms. But they were able to confidently use and describe the number of components for each object and the number of ice cream scoops per container. Note that it is the invariant numerical relationship between object and component, or container and scoop, that is quantified by a rational number. From the children's responses, it appeared as if this numerical relationship may be founded on their initial awareness of the constitutive relationship between object and components (or container and contents), the necessary relationship for properly forming the object or filling the container. This prior knowledge could form an experiential foundation for the child's developing concept of rational number quantities.

\section{Changes in the concept of 'quantity' in rational number learning}

It is commonly accepted (see Verschaffel, Greer \& De Corte, 2007, for a review) that a young child's learning of whole numbers builds on the conception of a quantity as a completed count of discrete objects. The process by which this conceptualisation is developed has been extensively researched. When children use whole numbers to identify the numerator and denominator of a fraction, they will thus be working conceptually with two completed counts of discrete objects. In the classical introduction to fractions, these are: the completed count of discrete pieces in the whole and the completed count of discrete pieces making up the identified part. But the rational number is neither of these quantities. Rather, it quantifies the relationship between these two whole number quantities.

What is more, because of the density of the rational numbers, it is not possible to reduce this relationship to a single count. No matter what counting unit is chosen, infinitely many rational relationships would require a subdivision of this unit to be accurately quantified. Quantifying these relationships would then require two counts (one of them a count for the subdivision). For this reason, quantifying a rational relationship is intrinsically more complex than quantifying a count. To properly conceptualise rational number quantities, we thus need to change our view of 'quantity' from something relating only to single whole number counts, to something more suitable to rational numbers.

The Grade 4-7 research project involved regular weekly meetings, for the duration of the second and third terms,

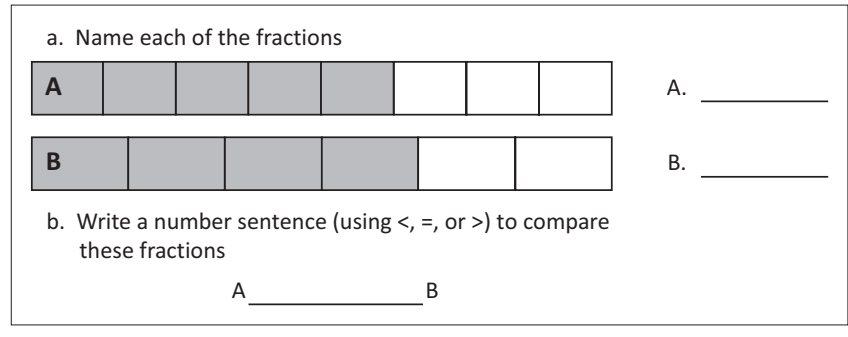

FIGURE 1: Example fraction task: What is being quantified?

with eight teachers who taught Grade 4-7 mathematics in the school. Teaching materials and samples of learners' work were collected for analysis. In the third term, teachers were individually interviewed and weekly Grade 4 classes were attended, in which the researcher was free to interact with the children. These teachers considered an important element of rational number learning to be the use of fractions to describe the size of the identified portion in a part-whole subdivision. To contribute to this learning, children were asked to complete worksheets containing tasks such as that shown in Figure 1. It was expected that the comparison relation in part (b) would be determined by a simple visual comparison of the shaded regions (valid because the wholes were the same size).

In answering this question, most children confidently counted the total number of subdivisions in each block, as well as the number of shaded subdivisions, to arrive at fractions (A: 5/8 and B: 4/6) describing each part-whole configuration. But in part (b) many children responded $A>B$, a relationship that would fit a comparison of counts of the total number of blocks and of the numbers of shaded (or unshaded) blocks. In the following question, where the comparison was between two unit parts, many of these children had responded $1 / 8>1 / 6$. When it was mentioned to these children that part (b) was asking them to use the fraction numbers to describe what they saw (which looked bigger) they immediately and confidently changed the relation to $\mathrm{A}<\mathrm{B}$.

Without any further prompting, many also changed their following response to $1 / 8<1 / 6$. It appeared from this interaction that the children's initial response was because they did not understand that part (b) was asking for a simple visual comparison. That is, that they were not clear that the purpose of part (b) was to show one way of making sense of the fraction quantities constructed in part (a). Rather, they seemed to see this task as asking them to use their understanding of whole numbers as counts, and the relational signs as describing order between whole numbers, to compare these composite fraction symbols. Once it became clear to them that the comparison was not of the counts, but of a different (visual) comparison of size, they confidently and correctly completed the worksheet.

Once the orientation of the task had been clarified, the comparison task helped the children realise that fraction quantities were not the same as simple counts. Most of the class readily came to this realisation. But the comparison did 
depend on the fact that in each question, the wholes were the same size, a constraint that was not clearly specified in the task. Thus, the importance of the whole was downplayed in this task. In the same research project, a different teaching sequence was observed that brought in the size of the whole for consideration. It was interesting that the children did not appear to engage readily with this aspect of the task.

This teaching sequence involved concrete experiences of sharing, as related to fractions. In the first two lessons, the teacher grouped the children into twos, threes or fours and asked them to share some given food items (such as 3 hot dogs or 1 cookie) fairly amongst the group. The children took great care to ensure that the portions were all equal. They were also able to confidently use fractions to describe the shares, showing familiarity and competence with fraction notation. In the third lesson, the class was grouped into pairs and each pair was given an apple to share. In preparing the lesson, the teacher had deliberately chosen apples of different sizes for sharing. The children divided their apples quite accurately into halves and reported that each had received a half of their apple. Then the teacher asked if all the children had the same amount of apple. The initial response of the children was that they did: they all had half an apple. When asked to physically compare their halves they noticed the difference in size, but they found this difficult to reconcile with the fact that they had shared fairly in their pairs to each get half an apple. The teacher explained that the original apples were of different sizes and so the halves would be different, but this explanation did not appear to dispel the confusion. The fair sharing in pairs, together with the fact that each had the same fraction of an apple appeared to outweigh the explanation given after the sharing, that the whole apples were initially different sizes.

We were left with the question of whether the effect of the whole may have been more readily acknowledged if the children had been explicitly asked to compare the sizes of the wholes before the sharing and if the aim of the activity had been presented as 'looking to see what would happen if different size wholes were shared'. Later work with individual children based on a supplementary task developed to explore the relational theme suggested that children may naturally identify the size of the objects being halved as a reason for halves being different sizes. The task given was:

James has a chocolate. He gives half to Peter. He gives half of what is left to Bob. Are these two halves the same?

Here the immediate response was that these halves were not the same. The reason given was that, for Bob, what was divided was smaller.

These interactions suggest that, with suitable activities, Grade 4 children may come to see rational number quantities as related to the amount in a part, rather than a simple count of pieces. Also that they may come to understand that the size of these amounts varies with both the rational number describing the amount and the size of what is considered as the whole. Three important components may be identified in this relationship: the part amount, the whole amount and the comparative rational number. Activities suitable for the Grade 4 child may allow the investigation of the effect of changing any single element on another, whilst holding the third fixed. It is questionable whether learners of this age would be able to effectively engage with the effects of covariation of two elements; this would require considerably more sophistication in thinking than would be expected of a Grade 4 child.

\section{Teachers and the relational nature of rational numbers}

A relational understanding is also useful for teachers, allowing more flexible and appropriate interpretations of children's responses. An example of this is provided in the teacher's interpretation of answers (shown in Figure 2) to the following question in a Grade 5 class test:

Use drawings to show how you would share 5 slices of bread among 2 people. What fraction would each person get?

The first two responses were unsurprising, but the third was different. Nonetheless, the teacher suspected that the child may have appropriately thought through the question. After the test, she asked the child to clarify the response. The child explained that each slice was cut into two equal parts and five of these ten pieces were then given to each person. So each person got 5/10 of the bread. In this response, the fraction describing each person's share was obtained by comparing the share with the total amount of bread. This is in contrast to the other two, which compared the share to one slice of bread. Here the rational number describing the share changes, depending on the choice of reference unit. It describes the relationship between the share and the chosen unit. That is, the rational number is a relative quantity and the chosen reference unit needs to be known in order for the absolute magnitude of the share to be known. Understanding this relationship allowed the teacher to respond appropriately to the child's answer, resulting in a positive learning experience.

\section{The relational nature of rational numbers in repeated subdivisions}

The importance of relative comparisons in the early learning of rational numbers raised the question of whether such comparisons may be important in the developed adult

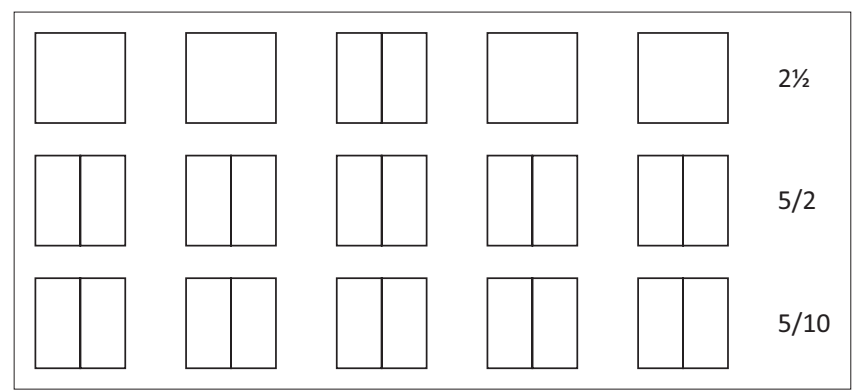

FIGURE 2: Responses to sharing five slices of bread between two people. 
concept. To further explore this question, a further research project was undertaken, in which a number of simple rational number tasks were administered first to five teachers and later to 22 student teachers. The participants were observed as they worked on these tasks. Their responses are discussed in this section.

In tasks relating to repeated subdivision, adults did appear to relate fraction quantities to a reference whole, although this whole was not always appropriately chosen. They were appropriate in adults' responses to the simple question described before:

James has a chocolate. He gives half to Peter. He gives half of what is left to Bob. Are these two halves the same?

Here the immediate response was ' $\mathrm{No}^{\prime}$ : Peter received more because he received half of the whole chocolate, whilst Bob received half of a half. Many adults immediately added that Bob received a quarter of the chocolate. Converting the representation of Bob's amount to a fraction of a whole allowed a direct comparison of the quantities as parts of the same whole. Others did not, identifying instead the different sizes of what was subdivided. With prompting, these adults made the conversion and quantified the comparison.

However, when asked to work with repeated subdivisions in a more abstract task, many adults did not choose appropriate reference units. Adults were asked to draw each of the fraction quantities in the repeated subdivision: $2 / 3$ of $3 / 4$ of $1 / 2$ and then to draw the final amount. The response of all the adults (both teachers and students) was to separately draw each of these fractions as parts of a whole of the same size and shape. To draw the final amount, some of the adults (mainly Grade 9-12 teachers) drew a single diagram showing the successive subdivision of a single whole, successfully identifying the composite quantity. To enable the other adults to draw the final amount, it was generally necessary to draw their attention to the repeated nature of the subdivision as indicated by the term 'of'.

\section{A further task was the following puzzle:}

You make three inventions to improve the fuel efficiency of a machine. One saves $30 \%$ of fuel, a second saves $45 \%$ and a third saves $25 \%$

How much fuel can you save if you use all three inventions at once?

In their first approach to this puzzle, all the adults first added the percentages together to come to a combined saving of $100 \%$. They then noted that a $100 \%$ savings was not practically possible and many looked for alternative approaches. Some, however, saw this as demonstrating the difference between mathematics and the 'real world' and were happy to say that $100 \%$ gave a mathematical solution that was unrealistic. Those who searched for an alternative approach generally resorted to some sort of averaging procedure. One group of adults was given this question immediately after the chocolate subdivision question and they were told that these problems could be usefully related. But this had very little effect on their solutions. This group were then prompted to see the inclusion of the inventions as a process in which one was included after the other, and to calculate the savings accordingly. In response, half of the group appropriately calculated composite proportions of fuel use $(70 \%, 55 \%$ of $70 \%$ and $75 \%$ of $55 \%$ of $70 \%)$. The remainder needed a second prompt: asking what the initial fuel use was before the second invention was included. Once the process of finding cumulative proportions became clear, the adults responded convincingly to the puzzle (with varying degrees of calculational efficiency).

These responses indicated that, for both abstract and situated repeated subdivision tasks, adults naturally interpreted the rational quantity in relation to a reference unit. But, unless they were clearly and explicitly stated as being different, the reference unit was taken as the same for each rational quantity in the task. This made abstract repeated subdivisions rather confusing. These results suggest that a relational comparison may indeed be part of adults' conception of rational numbers, but that the reference unit may generally be standardised, resulting in a lack of flexibility in the relational use of this concept.

\section{The number line: Magnitude as a relational concept Identity and embedding}

The observation that the property of magnitude unites whole and rational numbers is fundamental for the integrated theory of Siegler et al. (2011). But deducing from this that there is no fundamental conceptual change in the child's transition from whole number thinking to rational number thinking depends on a conflation between the ideas of identity and of embedding. For magnitude is indeed a common property of these numbers, when viewing both whole numbers and rational numbers as embedded in the real number field. But the child, who has no conception of real numbers, does not view these numbers as real numbers and so this may not be a common property when viewed in terms of conceptual development. To be precise, this is a common property of the images of whole and rational numbers under the respective embeddings of the whole and rational number structures into the real number field. This configuration of embeddings is represented diagrammatically in Figure 3.

An identification of a mathematical object with its image under an embedding is common in axiomatic mathematical practice. In this case, the embedding allows the domain to inherit the additional structure of the range space. But, when investigating conceptual development, this inherited structure originates in the additional conceptual structure of the range, not in the original conceptual structure of the domain. 


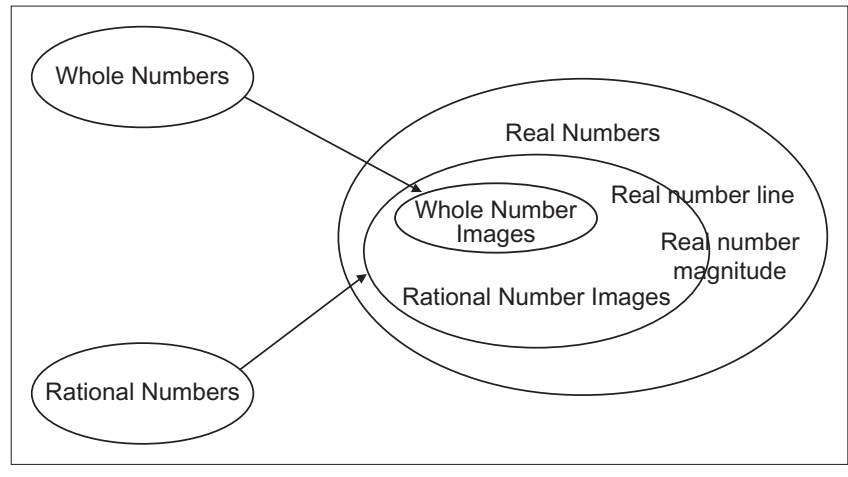

FIGURE 3: Embedding whole and rational numbers in real numbers.

The property of magnitude that unifies whole and rational numbers is the capacity of these quantities to be represented by a point on the real number line. This is a property of the images of these numbers when embedded in the real numbers. But the question remains whether this is a property of these number concepts before they are subsumed in the development of the later and richer real number concept. This question may be answered by the investigation of the relational nature of the number line as a model of quantity.

\section{The relational nature of rational numbers and the number line}

To investigate adults' use of the number line as a conceptual model for rational numbers, students and teachers were presented with a blank number line marked only with a zero point and asked:

Where can you place $15 / 4$ on the given number line?

In each case, the respondent's first action was to mark ' 1 ' as a unit on the line. The immediacy with which this was carried out suggested that this action was an automatic first response to the number line task. Once the unit had been marked, adults determined the approximate position of $15 / 4$, based on the distance between 0 and 1 . When asked if $15 / 4$ could have been placed anywhere else, they replied ' $\mathrm{No}^{\prime}$.

The observation that in each case, the unit (1) was placed first, and that this placement was immediate, suggests that a reference interval, such as [0.1], may be an implicit component of the number line that forms a conceptual model for rational numbers. The placement of the unit identifies an interval that determines the scale of the line and this uniquely determines the position of any other number on the line. It may be noted that, even though the research participants each placed a ' 1 ' as the unit, the scale would have been determined by the placement of any non-zero number on the line.

In a second query, I called their attention to the fact that they had first placed the 1 and asked where they could place the $15 / 4$ before the 1 was placed. Generally, the response to this was 'Anywhere'. These adults were thus aware that a number line with a zero but no reference interval (or scale) did not fully define absolute magnitude: the 15/4 could then be placed anywhere. The placement of a magnitude as a point on the line only becomes absolute once a single reference quantity has been placed, through the need to maintain the correct rational relationship with the reference quantity.

The need for a reference unit to fully specify the point for any number on the line (even a whole number) suggests that this conceptual model of magnitude is a relational (relative) model, rather than an absolute model. That is, working with whole or rational numbers as magnitudes uniquely placed on the number line will require a conceptual transition from numbers as absolute counts, to numbers as relational, in this case relative to the chosen reference unit. This is the same transition that this article argues is fundamental for the child's learning of rational numbers. Taking magnitude to be fundamental to the abstract concept of rational numbers thus implies that the relational property is fundamental to the concept of a rational number, even in the abstract.

As a further observation, the immediate placement of ' 1 ' as a unit by the adult participants suggests that the reference unit is an implicit aspect of the concept that is also generally standardised. That is, flexibility in the choice of the reference unit may not be a well-developed aspect of the rational number concept. This is supported by the observation that adults first responded as if the position of the number 15/4 was fully determined and also by the observation that common diagrams of fractions in repeated subdivisions, such as $2 / 3$ of $3 / 4$ of $1 / 2$, showed each fraction as a part of a whole of the same size. In both cases, when the possibility of changing the reference unit was brought to their attention, adult participants were able to usefully engage with the flexible, relational nature of the rational number. But this flexible relational engagement generally needed to be prompted.

\section{Possible implications for teaching}

This view of the conceptual change between whole and rational numbers has a number of implications for teaching. An important change at the outset would be to strengthen and build on the informal relational comparison scheme. Then, as the child's whole number competence grows, to develop ways to quantify these comparisons by relating counts, in this way deliberately developing rational numbers as a level of abstraction above that of whole numbers. The use of activities involving the measurement sub-construct and unit fractions as a means to quantify subdivision would also contribute to this development. The Grade $\mathrm{R}$ research project included the investigation of a number of such activities at the Grade $\mathrm{R}$ level. This will be reported in a subsequent article. Also, as is evident in the discussion of adults' use of the number line, using number lines and teaching approaches building on magnitude will also contribute to developing this relational understanding. Another explicit focus that may prove valuable for the rational number transition would be the move to relative, rather than absolute quantities: looking at fractions as 'how much of the whole' (relative) rather than 'how much in total' (absolute). 
In the work with children in these projects, and particularly in the work at Grade 4 level, it appeared as if acknowledging the fundamental difference between whole numbers as counts and rational numbers as relational comparisons may be a powerful lever for helping the child to achieve the transition to rational number thinking. In a number of instances, discussing whole numbers and rational numbers as different but related concepts seemed to make it easier for the child to consider whole and rational numbers alongside each other and so explore their consonances and dissonances to learn the interrelations between these two concepts. From a motivational perspective, acknowledging this difference when learning rational numbers allowed the child to attribute differences in properties of whole and rational numbers to differences between the types of numbers being considered, rather than to their own misunderstanding of number. As a result, children appeared to have more control and be more confident as they navigated this conflicting conceptual process. Further research related to this issue may prove valuable for the practice of teaching.

\section{Conclusion}

The rational number conceptual field shows more complexity than the whole number field, both in the scope of application of rational numbers (relating in different ways to a broader range of phenomena) and in the level of technical proficiency required to master the symbol systems of this field. This article argues for a third increase in complexity: that rational numbers are fundamentally relational in nature and that the move from absolute counts to relative comparisons requires a further level of abstraction in our understanding of number and quantity. That is, that the conceptual development from whole numbers to rational numbers does not simply involve the 'filling in' of the set of possible values for quantities (to form a dense, although not yet complete, set). Rather it involves a change in understanding of what a quantity is (from an absolute count to a relative measure) and a corresponding change in understanding of how a quantity may be measured.

This argument is based on an in-depth analysis of data from four small-scale qualitative research projects. Such an approach allows the development of deep insight into the particulars of the learning situation, but the conclusions reached are grounded only in the data of the particular situation. To increase the empirical base of these conclusions, further research would be necessary. In particular, further investigation of the relational nature of the number line for indicating magnitude will be important. Also, teaching experiments designed to investigate teaching that explicitly acknowledges the difference between the nature of counting and rational numbers would be fruitful.

\section{Acknowledgements Competing interests}

The author declares that he has no financial or personal relationships that may have inappropriately influenced him in writing this article.

\section{References}

Behr, M., Lesh, R., Post, T., \& Silver, E.A. (1983). Rational number concepts. In R. Lesh, \& M. Landau (Eds.), Acquisition of mathematical concepts and processes (pp. 91-126). New York, NY: Macmillan.

Cortina, J.L., Visnovska, J., \& Zuniga, C. (2014). Unit fractions in the context of proportionality: Supporting students' reasoning about the inverse order relationship. Mathematics Education Research Journal, 26, 79-99. http://dx.doi. org/10.1007/s13394-013-0112-5

Gelman, R., \& Williams, E. (1998). Enabling constraints for cognitive development and learning: Domain specificity and epigenesis. In D. Kuhn, \& R. Siegler (Eds.) Cognition, perception and language. Handbook of child psychology (Vol. 2; 5th edn.; pp. 575-630). New York, NY: Wiley.

Kieren, T.E. (1976). On the mathematical, cognitive, and instructional foundations of rational numbers. In R. Lesh (Ed.), Number and measurement: Papers from a research workshop (pp. 101-144). Columbus, OH: ERIC/SMEAC.

Kieren, T.E. (1988). Personal knowledge of rational numbers: Its intuitive and formal development. In J. Hiebert, \& M. Behr (Eds.), Number concepts and operations in the middle Grades (pp. 162-181). Reston, VA: National Council of Teachers of Mathematics.

Kilpatrick, J., Swafford, J., \& Swindell, B. (Eds.). (2001). Adding it up: Helping children learn mathematics. Washington, DC: National Academy Press.

Lamon, S.J. (2007). Rational numbers and proportional reasoning. In F.K. Lester Jr. (Ed.), Second handbook of research on mathematics teaching and learning: A project of the national council of teachers of mathematics (pp. 629-667). Charlotte, NC: Information Age Publishing.

McMullen, J., Hannula-Sormunen, M., \& Lehtinen, E. (2013). Young children's recognition of quantitative relations in mathematically unspecified settings. The Journal of Mathematical Behavior, 32, 450-460. http://dx.doi.org/10.1016/j. jmathb.2013.06.001

McMullen, J., Hannula-Sormunen, M., \& Lehtinen, E. (2014). Spontaneous focusing on quantitative relations in the development of children's fraction knowledge. Cognition and Instruction, 32(2), 198-218. http://dx.doi.org/10.1080/07370008. 2014.887085

McMullen, J., Laakkonen, E., Hannula-Sormunen, M., \& Lehtinen, E. (2015). Modeling the developmental trajectories of rational number concept(s). Learning and Instruction, 37, 14-20.

Moss, J., \& Case, R. (1999). Developing children's understanding of the rationa numbers: A new model and an experimental curriculum. Journal for Research in Mathematics Education, 30, 122-147. http://dx.doi.org/10.2307/749607

$\mathrm{Ni}$, Y., \& Zhou, Y.D. (2005). Teaching and learning fraction and rational numbers: The origins and implications of whole number bias. Educational Psychologist, 40(1) 27-52. http://dx.doi.org/10.1207/s15326985ep4001_3

Siegler, R.S., Fazio, L.K., Bailey, D.H., \& Zhou, X. (2013). Fractions: The new frontier for theories of numerical development. Trends in Cognitive Sciences, 17(1), 13-19. http://dx.doi.org/10.1016/j.tics.2012.11.004

Siegler, R.S., Thompson, C.A., \& Schneider, N. (2011). An integrated theory of whole number and fractions development. Cognitive Psychology, 62, 273-296. http:// dx.doi.org/10.1016/j.cogpsych.2011.03.001

Vanvakoussi, X., \& Vosniadou, S. (2004). Understanding the structure of the set of rational numbers: A conceptual change approach. Learning and Instruction, 14 453-467. http://dx.doi.org/10.1016/j.learninstruc.2004.06.013

Verschaffel, L., Greer, B., \& De Corte, E. (2007). Whole number concepts and operations. In F.K. Lester Jr. (Ed.), Second handbook of research on mathematics teaching and learning (pp. 557-628). Charlotte, NC: Information Age Publishing, teaching.

Wright, V. (2014). Towards a hypothetical learning trajectory for rational number. Mathematics Education Research Journal, 26(3), 635-657. http://dx.doi. org/10.1007/s13394-014-0117-8 